\title{
OBSERVATIONS OF GASEOUS DISKS IN CLOSE BINARIES
}

\author{
B. W. B A L DW IN
}

University of Victoria, Department of Physics, Victoria, B.C., Canada

\begin{abstract}
Recent observations of emission lines from circumstellar disks in Algol-type binaries have revealed new facts concerning their structure and evolution. In some cases circumstellar disks are unstable, but the nature of the instability is not known. For U Cep, the emission lines observed during the 1974 outburst can be interpreted as arising from an expanding shell of gas about the primary. Other systems which have displayed emission line variability are RW Tau, SW Cyg, W Del, AQ Peg, and VW Cyg. There is also some evidence to suggest that RYGem has experienced an outburst similar to that of U Cep. Emission lines observed in the eclipse spectrum of RY Gem by McKellar in 1947 were greatly different in strength and profile from those observed recently and cannot be interpreted as arising from a circumstellar disk rotating about the primary.
\end{abstract}

